\title{
Competencias digitales de estudiantes y profesores universitarios: una revisión sistemática
}

\author{
Edith Solórzano Chaca \\ esolorzano2012ch@gmail.com \\ https://orcid.org/ 0000-0002-8376-4473
}

\section{RESUMEN}

El objetivo de esta revisión sistemática fue conocer las competencias digitales de los docentes y estudiantes universitarios en las dimensiones de aprendizaje, informacional, comunicativa, cultura digital y tecnológica. La investigación se realizó siguiendo la metodología PRISMA extrayendo los artículos de las bases de datos como EBSCO, Scopus, Springer, Scielo y ProQuest en un mes. Los procesos de identificación, cribado, elegibilidad e inclusión dudaron mes y medio. Se consideraron 24 investigaciones de un total de 236. Entre los criterios de inclusión fueron: artículos indexados de los años 2019 y 2020, que traten de las competencias digitales de estudiantes y docentes universitarios. Los criterios de exclusión, se consideró aquellos documentos que no fueron revisados por pares, que evidencien deficiente metodología o que se salían del tema de la investigación. Los resultados indican que en la Era Digital en que se encuentra la educación universitaria se requiere la consolidación del uso de las herramientas tecnológicas por parte de los docentes y estudiantes para lograr mejores resultados en el proceso de enseñanza aprendizaje y sobre todo en el campo de la investigación científica.

Palabras clave: competencia digital, aprendizaje, informacional, comunicativa, cultura digital, tecnológica. 


\title{
Digital competences of university students and teachers: a systematic review
}

\begin{abstract}
The objective of this systematic review was to know the digital competences of university teachers and students, in the dimensions of learning, informational, communicative, digital and technological culture. The research was carried out following the PRISMA methodology, extracting articles from databases such as EBSCO, Scopus, Springer, Scielo and ProQuest in one month. The identification, screening, eligibility and inclusion processes lasted a month and a half. 24 investigations out of a total of 236 were considered. Among the inclusion criteria were: indexed articles from the years 2019 and 2020, which deal with the digital competences of university students and teachers. The exclusion criteria were those documents that were not peer-reviewed, that show poor methodology or that went outside the research topic. The results indicate that in the Digital Era in which university education finds itself, the consolidation of the use of technological tools by teachers and students is required to achieve better results in the teaching-learning process and especially in the field of scientific investigation.
\end{abstract}

Keywords: digital competence, learning, informational, communicative, digital culture, technology.

Artículo recibido: 02 noviembre. 2021 Aceptado para publicación: 28 noviembre 2021 Correspondencia: esolorzano2012ch@gmail.com

Conflictos de Interés: Ninguna que declarar 


\section{INTRODUCCIÓN}

En la Era digital los jóvenes universitarios deben demostrar un dominio de las competencias digitales, las universidades brindar la enseñanza y aprendizaje en aulas taller implementadas con TIC y los catedráticos demostrar una formación en habilidades digital. Por ello, es importante que los docentes utilicen recursos digitales para discriminar los datos, crear contenido digital, compartir la información, usar las redes sociales en el aprendizaje y, a la vez, participar en proyectos de colaboración en línea. La preparación del docente universitario en habilidades tecnológicas permitirá formar estudiantes que practiquen la ciudadanía digital en la sociedad en red.

El Marco Común de Competencia Digital Docente, en la versión de octubre del 2017, menciona que existen cinco competencias digitales que todo docente debe desarrollar y acreditar para mejorar su práctica pedagógica y perfeccionamiento continuo en la sociedad del siglo XXI, tales competencias son la información y alfabetización informacional, la comunicación y colaboración, la creación de contenidos digitales, la seguridad y, finalmente, la resolución de problemas. Bajo estos requerimientos se formula las siguientes interrogantes, ¿las universidades están respondiendo a las necesidades de la educación actual? ¿Los catedráticos demuestran y acreditan las habilidades digitales para el cargo? y, por último, ¿los estudiantes aprenden y demuestran habilidades digitales?

La pandemia ha puesto en evidencia el dominio de las competencias digitales de estudiantes y docentes universitarios que tuvieron que migrar de la presencialidad a la virtualidad en el desarrollo de las sesiones de aprendizaje. Los estudiantes se vieron en la imperiosa necesidad de recibir las clases por plataformas digitales para realizar trabajos colaborativos sincrónicos y asincrónicos. En el caso de los docentes adaptaron su cátedra, en un corto tiempo, al modo virtual preparando materiales y una serie de secuencias didácticas donde se emplean las plataformas con chat, foro y videoconferencias para responder a la educación virtual. Esa transformación digital fue angustiante y tediosa en un inicio, pero al transcurrir los meses se fueron adaptando a la metodología virtual y sus características.

Los docentes universitarios tuvieron que recibir capacitaciones para el uso de las plataformas como el Zoom, Moodle y otros; en el caso de los estudiantes universitarios, recibieron cursos extracurriculares para usar herramientas digitales de búsqueda, 
procesamiento de la información y trabajo colaborativo remoto. A pesar de las capacitaciones, aún se observa que las competencias digitales de docentes y estudiantes no están respondiendo a las presentes circunstancias. Por ello, esta investigación tiene como objetivo conocer las competencias digitales de los docentes y estudiantes universitarios, en especial, en las dimensiones de aprendizaje, informacional, comunicativa, cultura digital y tecnológica.

Al observar el presente desafío digital en el contexto de pandemia, se plantea como propósito de esta revisión sistemática conocer las competencias digitales de los estudiantes y docentes universitarios.

\section{METODOLOGÍA}

Teniendo en cuenta la Declaración PRISMA, se efectúo una revisión de las bases de datos EBSCO, Scopus, Springer, Scielo y ProQuest, entre los días 10 de noviembre hasta el 20 de diciembre del 2020, aplicándose las fases de identificación, cribado, elegibilidad e inclusión de la declaración Prisma durante un mes. Entre los términos de búsqueda se utilizó las palabras claves en idioma español e inglés como "digital competence", "learning", “informational”, “communicative”, "digital culture”, “technology", obteniendo un total de 236 documentos y siendo seleccionados 24 investigaciones. Entre los criterios de inclusión fueron: artículos indexados de los años 2019 y 2020, que traten de las competencias digitales de estudiantes y docentes universitarios. Y entre los criterios de exclusión, se consideró aquellos documentos que no fueron revisados por pares, que evidencien deficiente metodología o que se salían del tema de la investigación. 


\section{RESULTADOS Y DISCUSIÓN}

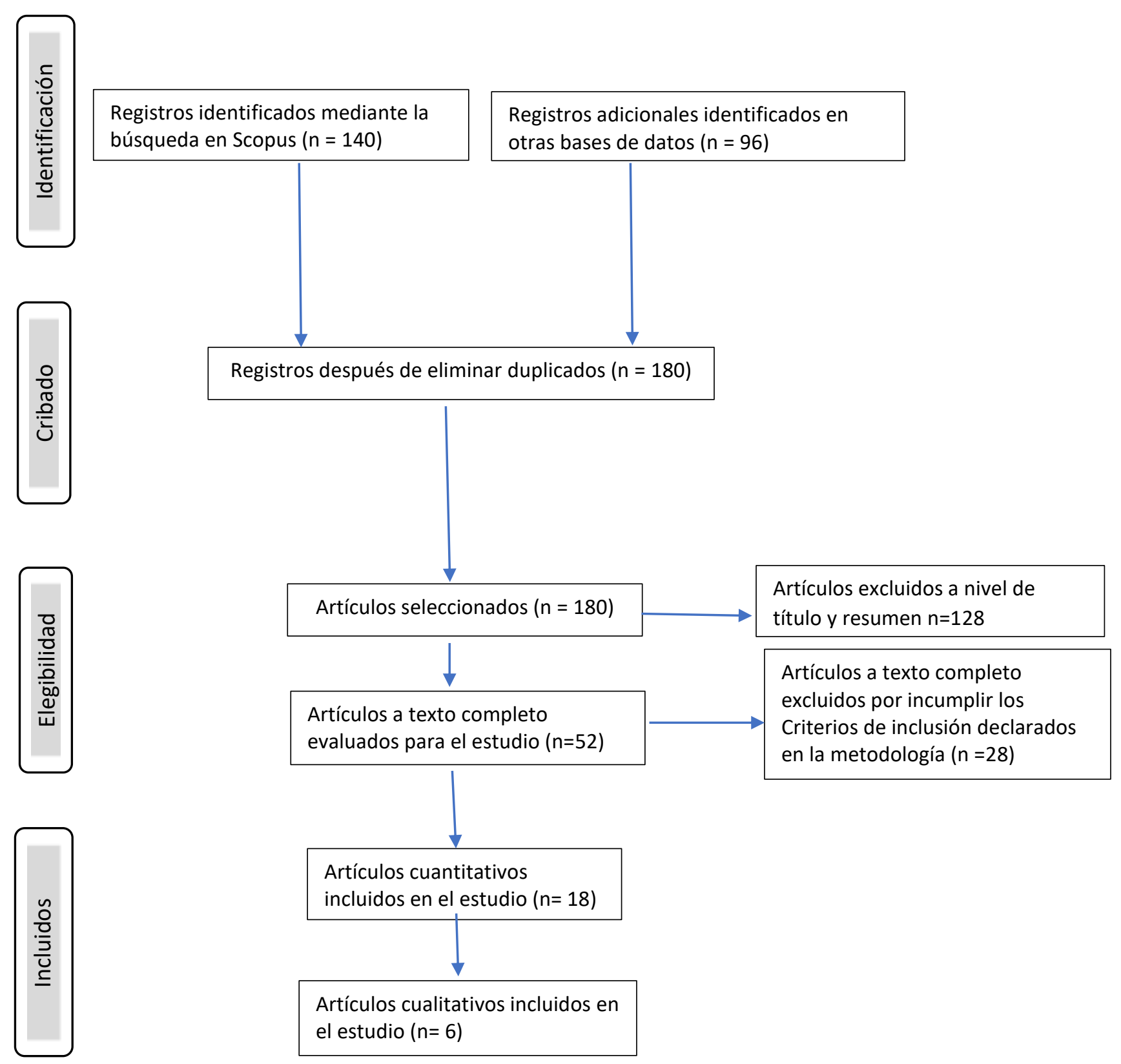

Figura 1. Adaptación de PRISMA Flow Diagram (Moher, Liberati, Tetzlaff,

\& Altman, 2009) 
Tabla 1. Artículos que cumplen los criterios de inclusión

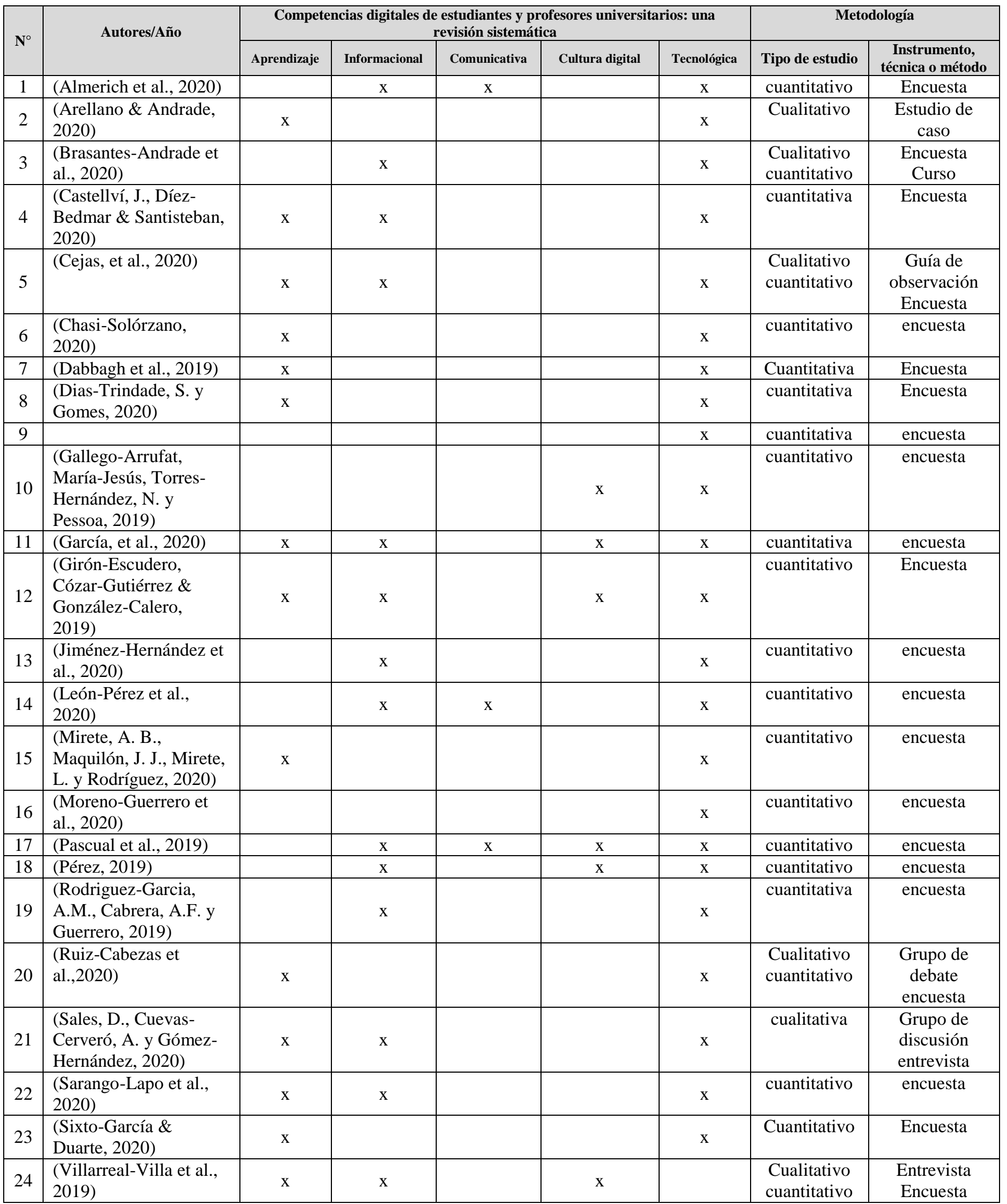


De las 24 investigaciones, el 58,33\% responde a la dimensión aprendizaje, el 58,33\% a la informacional, un $12,5 \%$ a la comunicativa, el $25 \%$ a la cultura digital y un $95,83 \%$ a la tecnológica. Se evidencia que las dimensiones se relacionan entre ellas. Con respecto a los tipos de estudio, el $25 \%$ fueron de enfoque cualitativo y un $75 \%$, cuantitativo.

Se observa que aprendizaje, informacional, comunicativa, cultura digital y tecnológica son dimensiones fundamentales en las competencias digitales de los estudiantes y docentes universitarios que se discuten a continuación.

\section{DISCUSIÓN}

En relación al aprendizaje y la transformación de la información en conocimiento, el uso de las Tecnologías de la Comunicación e Información (TIC) en las sesiones de aprendizaje constituyen una oportunidad educativa porque ponen a disposición de la enseñanza universitaria una serie de recursos digitales. Los docentes sientes que existen posibilidades en el uso de estas herramientas y una mejora en los procesos de enseñanza y aprendizaje (Ruiz-Cabezas et al., 2020). Es necesario advertir que, en el contexto actual de pandemia por COVID-19, se necesita que los gobiernos impulsen políticas acordes a la situación que se da en el campo de la educación cuyo objetivo debe ser fortalecer la infraestructura digital y la conectividad en las universidades; asimismo, que los docentes sean capacitados al más alto nivel en el desarrollo de competencias sobre gestión de entornos virtuales.

De la misma forma, Arellano y Andrade (2020) sostienen que los docentes reflexionan sobre los usos de las herramientas digitales en sus actividades cotidianas, por ejemplo, en las sesiones de aprendizaje y en la elaboración de materiales virtuales de la universidad. Las aulas se convierten en espacios donde los estudiantes transforman la información en conocimiento. El docente reflexiona acerca de su labor educativa; y más aún, en el contexto en que vive, en pleno desarrollo de la Era Digital, no es un paria de la tecnología, su necesidad lo ha hecho incursionar en ella, sin embargo, ha entendido que las debilidades de la conectividad, no es traducido como la brecha digital, sino que envuelve diversos elementos inmersos desde la aplicación de software y la modernidad constante del hardware, se puede decir que tener un celular y las oportunidades de conectividad en línea, desde la perspectiva educativa debe estar la servicio de la educacion, no necesariamente de la educacion lectiva, sino de contenidos que permitan iniciar la creatividad del estudiante desde los primero años de escolaridad. La actual coyuntura deja 
un elemento enriquecedor en lo que corresponde a la dinámica del uso de la tecnología al servicio de la educacion.

Sin embargo, un error que se observa en la práctica docente es la priorización del uso las TIC como medio de información dejando de lado su empleo en la investigación. Existen universidades con una variedad de aplicaciones, acceso a las herramientas tecnológicas e internet que los docentes universitarios tienen a su disposición, pero solo las usan como medio de información y comunicación, olvidando los beneficios de estos en la investigación (Cejas et al., 2020). Se debe considerar a las TICs como un instrumento para la innovación y realización de proyectos académicos en entornos virtuales. Los docentes universitarios, muchas veces, publican en la red, crean contenidos digitales, pero presentan dificultades en el perfil de investigador (Pérez, 2019). El acceso a las herramientas tecnológicas en la Era Digital, su uso y manejo, no solo debe limitarse como medio de comunicación; sino, debe constituirse como una puerta de acceso al mejor contenido cultural, artístico y científico, razón a ello, en la actualidad, hay grandes corporaciones educativas y universidades que desarrollan programas de posgrado de manera virtual o remota con grandes beneficios académicos y laborales para sus estudiantes, fortaleciendo la tesis que la educación virtual es igual o mejor que la educación presencial.

Por otro lado, los universitarios consideran que las herramientas y los entornos digitales brindan una ventaja en sus aprendizajes; inclusive, ellos valoran estas tecnologías (Dabbagh et al., 2019) porque les permite incrementar y complementar sus habilidades digitales adquiridas en experiencias educativas anteriores. El estudiante universitario optimiza los recursos de aprendizaje, a pesar de algunas limitaciones en las competencias digitales. En cambio, el docente universitario presenta dificultades en la planificación y aplicación de recursos tecnológicos dificultando su rol de mediador en el aprendizaje (Chasi-Solórzano, 2020). En la actualidad hay dos frentes: por un lado, docentes con limitaciones en el desenvolvimiento y manejo de entornos virtuales por el permanente desarrollo y creciente innovación de las TICs; y, por otro lado, estudiantes muy motivados y permanentemente digitalizado; es decir, con acceso directo a las últimas tecnología y entornos virtuales. Esta situación, muchas veces ha sido confrontacional, por lo que las autoridades universitarias han tenido que fortalecer las competencias de los docentes a 
través de programas de inducción, diplomados o talleres, de manera que el docente cumpla su rol de guía o mentor.

Por ello, urge que, en las universidades, los docentes sean preparados en habilidades digitales para clases sincrónicas y asincrónicas, la gestión de la información y del conocimiento, la preparación de material digital, la reflexión sobre su papel de investigador y mediador del aprendizaje. La preparación de material audiovisual complementa las asignaturas y contribuye al logro de las competencias programadas. Es necesario la preparación continua de los catedráticos en clases sincrónicas y asincrónicas (Sixto-García \& Duarte, 2020). Si se pasa de las clases presenciales a las sesiones virtuales o mixtas, el rol del docente mediador demanda una serie de destrezas para el desarrollo de las sesiones de clase donde la pertinencia del material digital es primordial. Por consiguiente, en la docencia el uso de las TICs se relaciona con los procesos de enseñanza y aprendizaje. Un docente es competente cuando entrelaza las intenciones y estrategias con su nivel de competencia digital (Mirete, Maquilón, Mirete, y Rodríguez, 2020) y su perfil bimodal (sincrónico y asincrónico) (Sarango-Lapo, 2020). Solo si el catedrático reconoce esas habilidades de pensamiento, de planificación y producción de material digital y ve en las TICs una oportunidad e innovación, estaremos hablando de un profesional acorde a las demandas de la educativa universitaria.

Cuando mencionamos la habilidad informacional, nos referimos a la obtención y evaluación de la información y su tratamiento en los entornos virtuales que deberían ser observables en los docentes y estudiante universitario; por eso, el uso de las TIC en proyectos académicos tiene resultados óptimos tanto en la adquisición de habilidades y aprendizajes. En los planes de estudio y curriculares se deben considerar de manera transversal el uso y aprovechamiento de las TIC (León-Pérez, Bas, \& Escudero- Nahón 2020). Las universidades que se preocupan en las habilidades digitales de sus docentes y estudiantes, incentivan la constante presentación de proyectos de investigación para ser publicados en revistas indexadas ganando una reputación dentro de la comunidad educativa y científica. Existe en la actualidad una preocupación por las habilidades investigativas y digitales de docentes y estudiantes universitarios para cubrir estos requerimientos digitales del siglo XXI a través capacitaciones docentes y módulos complementarios a la malla curricular de la carrera. 
Por eso, esas habilidades digitales como acceso, búsqueda, gestión y evaluación de la información y recursos digitales deben estar presentes en el perfil del docente universitario; sin embargo, se encuentran en proceso de desarrollo o nivel intermedio. Se necesita implantar oportunos planes académicos en materia de competencia digital (Rodriguez-Garcia, Cabrera, y Guerrero, 2019). Dichas capacitaciones deben ir de las más sencillas a las complejas, pasando por las habilidades de Tecnologías de la información y comunicación (TIC), las Tecnologías para el aprendizajes y conocimiento (TAC) hasta las Tecnologías para el empoderamiento y la participación (TEP) que permitan el desarrollo profesional de las competencias digitales de los docentes universitarios en orden de progresión y complejidad del componente de gestión (García et al., 2020). Es necesario una preparación y acreditación de las habilidades tecnológicas de búsqueda, validación, almacenamiento de la información y recursos digitales de los docentes universitarios a un nivel avanzado para su óptimo desenvolvimiento pedagógico y de investigador.

No obstante, tener habilidades digitales en niveles avanzados no es suficiente para un óptimo resultado de aprendizaje, sino poseer y hacer uso de las habilidades de pensamiento y creación de contenido digital. Los planes de estudios en TIC de los docentes de posgrado deben considerar la competencia crítica y la creación de contenidos digitales (Jiménez-Hernández et al., 2020) y, a la vez, un mayor compromiso en la renovación de la información de los materiales digitales que consumen los estudiantes universitarios (Villarreal-Villa et al., 2019). Esas destrezas digitales y la gestión del conocimiento deben ser transversales e impartidas desde los inicios de la escolaridad y puestas en práctica en la vida universitaria. La presencia de hábitos y habilidades técnicas sin una gestión y producción de la información acompañada de una carente competencia critica-reflexiva hace que los estudiantes presenten dificultades antes del ingreso a la universidad (Sales, Cuevas-Cerveró y Gómez-Hernández, 2020). En la actualidad toda universidad acreditada o licenciada posee una plataforma virtual como herramienta tecnológica de soporte académico para cada estudiante. Estas plataformas como parte de las TICs son de gran ayuda porque permite la comunicación interpersonal a través de diálogos, discusión y trabajo colaborativo donde se consume y produce materiales digitales logrando progresos en los aprendizajes de los estudiantes y grupos de trabajo; 
inclusive, presentan tutoriales y espacios de asesorías personalizadas para mejorar las habilidades tecnológicas y la creación de contenido digital.

De la misma manera, es necesario que los docentes universitarios posean un nivel óptimo en el desarrollo de contenidos digitales y el uso de entornos para que los estudiantes aprendan de ellos, y, a la vez, el compromiso de las universidades en la aplicación de planes de estudios sobre estos temas. Los futuros docentes presentan dificultades para redactar discursos críticos con la información de internet. Urge una programación universitaria que considere la interpretación de la información de los medios y una actitud crítica ante los problemas sociales (Castellví, J., Díez-Bedmar \& Santisteban, 2020). Toda universidad que alberga programas, escuelas académicas o facultades de educación, necesariamente en su malla curricular deben consignar el desarrollo de competencias relacionadas al desenvolvimiento en entornos virtuales generados por las TICs., ya que el sistema educativo y el desarrollo de la tecnología así lo demanda.

Con respecto a la habilidad comunicativa, la comunicación interpersonal y comunicación social juegan un papel decisivo en la puesta en práctica de las competencias digitales porque transversalmente desarrollan competencias de pensamiento complejo y trabajo colaborativo. Las competencias en TIC permiten alcanzar las competencias de alta habilidad. (pensamiento superior y trabajo en equipo) (Almerich et al., 2020). Estas habilidades reclaman de los docentes y estudiantes universitarios realizar trabajos en comunidades de aprendizajes y entornos virtuales donde comparten información y elaborar de manera colectiva tareas y proyectos de aprendizaje. El adecuado manejo de las TICs en educación, específicamente en el proceso de enseñanza aprendizaje es una palanca para lograr nuevos aprendizajes y conocimientos. Sus diferentes manejos pueden servir a cada estudiante en el desarrollo de la habilidad o aprendizaje que se quiere lograr. En relación a la cultura digital, las prácticas sociales y culturales de la sociedad de la información, la ciudadanía digital, la identidad digital y el derecho de lo que circula en la red son temas que tanto estudiantes y docentes universitarios deben aprender y respetar. El acceso y distribución de información debe darse por canales autorizados respetando el derecho de autor y dejando una huella digital responsable. La seguridad digital es una habilidad del profesorado para ser digitalmente competente, así como el conocimiento de las repercusiones de las tecnologías en el consumo y el medioambiente para ejercer una responsable ciudadanía digital (Gallego-Arrufat, Torres-Hernández y Pessoa, 2019). El 
almacenamiento, la distribución y la seguridad de la información son temas que se vienen aprendiendo como ciudadano digital. Sin embargo, en los estudiantes y docentes universitarios podemos observar desconocimiento de la gestión de la información, dificultades en el seguimiento de la huella digital y otros problemas informáticos (Pascual et al., 2019). Se considera que la educación universitaria actual tiene un pilar básico, la innovación de la Era Digital, cuyo protagonista es el docente, quien está llamado a convertirse el competente digital y para ello debe hacerse de las dimensiones como: manejo de información, comunicación en entornos virtuales, creación de contenidos virtuales, protección de datos y resolución de problemas; su desconocimiento le acarreará muchos problemas y limitaciones.

Por otro lado, la habilidad tecnológica, la alfabetización digital, el conocimiento y dominio de los entornos virtuales es una necesidad del ciudadano de la sociedad del conocimiento y, en especial, de los estudiantes y docentes universitarios. La competencia digital de los docentes es de un nivel moderado. Es necesario la capacitación en TIC para responder a las demandas de la universidad (Girón-Escudero et al.,2019). Por eso, urge capacitar a los docentes universitarios en diferentes ámbitos de su quehacer educativo, tanto en habilidades digitales, mediador del aprendizaje en entornos virtuales y curador de contenido digital. El nivel de la competencia digital, a veces, se vincula con la edad del catedrático. Se requiere reforzar el uso de la tecnología en diferentes realidades (Brasantes-Andrade et al., 2020). Para eso las capacitaciones en competencias digitales es una responsabilidad compartida por todos los agentes educativos: docentes, estudiantes y autoridades. Además, los cursos de capacitación en las áreas de la competencia digital para docentes universitarios deben conducir a la mejora de las habilidades tecnológicas y el desempeño docente (Dias-Trindade. y Gomes, 2020). La Era digital ha cambiado la forma de enseña y aprender en la virtualidad. El docente es testigo de los constantes cambios y debe adaptarse a estos; por ello, se ve en la imperiosa necesidad de utilizar metodología bimodales y herramientas en entornos digitales que muchas veces son elementos cotidianos en la vida de los estudiantes universitarios.

Finalmente, las habilidades en el uso de dispositivos informáticos de navegación y el mantenimiento de los equipos son habilidades digitales priorizadas en los estudiantes universitarios de la carrera de docencia; además, de adquirir una alfabetización básica para buscar, gestionar y evaluar la información y, finalmente, crear y compartir el 
contenido digital. Los estudiantes de docencia deberán compartir las habilidades tecnológicas que posean con sus futuros aprendices (Esteve-Mon, Llopis \& Adell-Segura, 2020). Las instituciones universitarias deben impartir la alfabetización informacional a los futuros profesores ya sea de manera transversal o a través de materias que desarrollen la competencia digital del estudiante (Moreno-Guerrero et al., 2020). La Era Digital exige que en la preparación del futuro docente se priorice los conocimientos, las habilidades y actitudes de las competencias digitales para hacer un uso responsable de la tecnología y estar a la altura de los retos de la educación actual.

\section{CONCLUSIONES}

Las competencias digitales de los docentes universitarios se encuentran en un nivel intermedio en el empleo de las herramientas tecnológicas en entornos virtuales y la producción de contenido digital provocando dificultades en el perfil del docente universitario.

En el contexto de la Era Digital en que se encuentra la educación universitaria se requiere la consolidación del uso de las herramientas tecnológicas por parte de los docentes y estudiantes para lograr mejores resultados en el proceso de enseñanza aprendizaje y, sobre todo, en el campo de la investigación científica.

El acceso a las herramientas tecnológicas en esta Era Digital, a nivel de pregrado y posgrado; su uso y manejo, no solo debe limitarse a ser un medio de comunicación; sino, que las plataformas virtuales de las universidades deben constituirse en una puerta de acceso al campo de la investigación científica.

Siendo una necesidad que los docentes hagan uso de las TIC para la mejora de los procesos educativos, se considera que toda universidad que alberga programas, escuelas académicas o facultades de educación, necesariamente en su malla curricular consignen el desarrollo de competencias relacionadas al desenvolvimiento en entornos virtuales generados por las TIC para formar a los futuros docentes de acuerdo a la Era Digital.

\section{REFERENCIAS BIBLIOGRÁFICAS}

Almerich, G., Suárez-Rodríguez, J., Díaz-García, I. y, \& Orellana, N. (2020). Structure of 21 st century competences in students in the sphere of education. influential personal factors. Educacion XX1, 23(1), 45-74. https://doi.org/10.5944/educxx1.23853

Arellano, A. y Andrade, R. A. (2020). Competencias digitales docentes en profesores 
universitarios. Journal Educational Innovation / Revista Innovación Educativa, 20(83),

http://eds.a.ebscohost.com/eds/detail/detail?vid=63\&sid=528eff2c-ce8d-4096b4ec-0fb4db523570\%40sdc-v-

sessmgr02\&bdata=Jmxhbmc9ZXMmc210ZT11ZHMtbG12ZQ\%3D\%3D\#AN=14 $5466529 \& \mathrm{db}=\mathrm{eue}$

Brasantes-Andrade, A. Cabezas-Gonzalez, M. y Casillas- Martin, S. (2020). Los nanoMOOC como herramienta de formación en competencia digital docente. Revista Ibérica de Sistemas e Tecnologías de Informação, 202-214.

https://search.proquest.com/docview/2452331341/9951D532AE634CA2PQ/15?account $\mathrm{id}=37408$

Castellví, J., Díez-Bedmar, M.-C. y, \& Santisteban, A. (2020). Pre-service teachers' critical digital literacy skills and attitudes to address social problems. Social Sciences, 9(8). https://doi.org/10.3390/SOCSCI9080134

Cejas, M. F., Lozada, B. N., \& Urrego A. J., Mendoza, D. J. y Rivas, G. (2020). The irruption of information and communication technologies (Ict), a challenge in the management of digital competences of university professors in Ecuador. RISTI Revista Iberica de Sistemas e Tecnologias de Informacao, 2020(37), 132-148. https://doi.org/10.17013/risti.37.132-148

Chasi-Solórzano, B. (2020). Integración de las TIC en los procesos de enseñanzaaprendizaje en la Facultad de Filosofía, Letras y Ciencias de la Educación de la Universidad Central del Ecuador. REIRE Revista d'Innovació i Recerca En Educació, 13(1), 1-18. https://doi.org/10.1344/reire2020.13.122235

Dabbagh, N., Fake, H. y, \& Zhang, Z. (2019). Student Perspectives of Technology use for Learning in Higher Education. RIED. Revista Iberoamericana de Educación a Distancia, 22(1), 127. https://doi.org/10.5944/ried.22.1.22102

Dias-Trindade, S. y Gomes, A. (2020). Digital teaching skills: Digcompedu checkin as an evolution process from literacy to digital fluency. Icono14, 18(2), 162-187. https://doi.org/10.7195/RI14.V18I2.1519

Esteve-Mon, F., Llopis, A. y, \& Adell-Segura, J. (2020). Digital competence and computational thinking of student teachers. International Journal of Emerging Technologies in Learning, 15(2), 29-41. 
https://doi.org/10.3991/ijet.v15i02.11588

Gallego-Arrufat, María-Jesús, Torres-Hernández, N. y Pessoa, T. (2019). Competence of future teachers in the digital security area. Comunicar, 27(61), 53-62. https://doi.org/10.3916/C61-2019-05

Garcia, D.A., Villarreal, J. E., Ortega, J.A., Cuellar, O.A. y Henao, C. F. (2020). Dimensiones de competencia digital en docentes universitarios: análisis relacional basado en componentes 1. Revista Ibérica de Sistemas e Tecnologias de Informação, 945-960. https://search.proquest.com/docview/2388305244/9951D532AE634CA2PQ/19? accountid $=37408$

Giron, V., Cózar, R. y Gonzalez-Calero, J. A. (2019). Analysis of self-perception on the level of teachers' digital competence in teachers training. Revista Electronica Interuniversitaria de Formacion Del Profesorado, 22(3), 193-218. https://doi.org/10.6018/reifop.373421

Jiménez-Hernández, D., González-Calatayud, V., Torres-Soto, A., Martínez, A. y, \& Morales, J. (2020). Digital competence of future secondary school teachers: Differences according to gender, age, and branch of knowledge. Sustainability (Switzerland), 12(22), 1-16. https://doi.org/10.3390/su12229473

León-Pérez, F., Bas, M., \& Escudero-Nahón, A. (2020). Self-perception about emerging digital skills in Higher Education students. Comunicar, 28(62), 89-98. https://doi.org/10.3916/C62-2020-08

Mirete, A. B., Maquilón, J. J., Mirete, L. y Rodríguez, R. A. (2020). Digital competence and university teachers' conceptions about teaching. A structural causal model. Sustainability (Switzerland), 12(12). https://doi.org/10.3390/SU12124842

Moher, D., Liberati, A., Tetzlaff, J., \& Altman, D. (2009). Preferred reporting items for systematic reviews and meta-analyses: The PRISMA statement. In BMJ (Online) (Vol. 339, Issue 7716, pp. 332-336). British Medical Journal Publishing Group. https://doi.org/10.1136/bmj.b2535

Moreno-Guerrero, A. J., Miaja-Chippirraz, N., Bueno-Pedrero, A. y, \& Borrego-Otero, L. (2020). The information and information literacy area of the digital teaching competence. Revista Electronica Educare, 24(3). https://doi.org/10.15359/REE.24-3.25 
Pascual, M. A., Ortega-Carrillo, J. A., Pérez-Ferra, M., Y., \& Fombona, J. (2019). Digital competences in the students of degree primary education teacher. Formacion Universitaria, 12(6), 141-150. https://doi.org/10.4067/S071850062019000600141

Pérez, R. (2019). Teaching digital competence in the teacher training institutes: Case of the Dominican Republic. Pixel-Bit, Revista de Medios y Educacion, 55, 75-97. https://doi.org/10.12795/pixelbit.2019.i55.05

Rodriguez-Garcia, A.M., Cabrera, A.F. y Guerrero, A. J. M. (2019). Competencia digital docente para la búsqueda, selección, evaluación y almacenamiento. Revista Interuniversitaria de Formación Del Profesorado, 33(3), 235-250. http://eds.a.ebscohost.com/eds/detail/detail?vid=24\&sid=528eff2c-ce8d-4096b4ec-0fb4db523570\%40sdc-vsessmgr02\&bdata=Jmxhbmc9ZXMmc210ZT11ZHMtbG12ZQ\%3D\%3D\#AN=14 $3353296 \& d b=$ fua

Ruiz, A., Medina, M., Pérez, E y Medina, A. (2020). University teachers' training: The Digital Competence. Pixel-Bit, Revista de Medios y Educacion, 58, 181-215. https://doi.org/10.12795/pixelbit.74676

Sales, D., Cuevas-Cerveró, A. y Gómez-Hernández, J.-A. (2020). Perspectivas sobre la competencia informacional y digital de estudiantes y docentes de Ciencias Sociales antes y durante el confinamiento por la Covid-19. https://doi.org/10.3145/epi.2020.jul.23

Sarango-Lapo, C. P., Mena, J., Ramirez-Montoya, M. y Real, E. (2020). La escala de Competencia Digital y uso de Recursos Educativos Abiertos (CD-REA): factores asociados a la competencia de los docentes universitarios bimodales. Revista Ibérica De Sistemas e Tecnologias De Informação, 545-558.

https://search.proquest.com/docview/2388305422/4FB4FEBD05234A16PQ/16?account id $=37408$

Sixto-García, J. y, \& Duarte, A. G. (2020). Self-destructive content in university teaching: New challenge in the digital competence of educators. Communication and Society, 33(3), 187-199. https://doi.org/10.15581/003.33.3.187-199

Villarreal-Villa, S., García-Guliany, J., Hernández-Palma, H. y, \& Steffens-Sanabria, E. (2019). Teacher competences and transformations in education in the digital age. 
Formacion Universitaria, 12(6), 3-14. https://doi.org/10.4067/S071850062019000600003 